\title{
Characterizing black hole metrics in quadratic gravity
}

\author{
A. Bonanno ${ }^{1,2}$ and S. Silveravalle ${ }^{3}$ \\ ${ }^{1}$ INAF, Osservatorio Astrofisico di Catania, via S.Sofia 78, I-9 5123 Catania, Italy \\ ${ }^{2}$ INFN, Sezione di Catania, via S. Sofia 64, I-95123, Catania, Italy \\ ${ }^{3}$ Dipartimento di Fisica G. Occhialini, Università degli studi di Milano Bicocca Piazza della Scienza, \\ 3, 20126 Milano, Italy
}

(Received 27 March 2019; published 24 May 2019)

\begin{abstract}
The recent discovery of non-Schwarzschild black hole spacetimes has opened new directions of research in higher-derivative gravitational theories. However, despite intense analytical and numerical efforts, the link with the linearized theory is still poorly understood. In this work we address this point for the EinsteinWeyl Lagrangian, whose weak field limit is characterized by the standard massless graviton and a spin-2 ghost. We show that the strength of the Yukawa term at infinity determines the thermal properties of the black hole and the structure of the singularity near $r=0$. Moreover, inspired by recent results in the asymptotic safety scenario we investigate the consequences of an imaginary ghost mass. In this case we find a countable set of solutions all characterized by spatial oscillations of typical wavelength determined by the mass of the spin- 2 field.
\end{abstract}

DOI: 10.1103/PhysRevD.99.101501

\section{INTRODUCTION}

Since the seminal work by Stelle [1] it has long been known that extensions of Einstein's gravitational theory containing $R^{2}$ and $C_{\mu \nu \sigma \tau} C^{\mu \nu \sigma \tau}$ operators in the Lagrangian are renormalizable in $d=4$ dimensions, but at the price of a loss of unitarity owing to a spin- 2 ghost. In recent times the possibility of defining the continuum limit around a non-Gaussian fixed point [2] has fueled new interest in this problem. Several authors have in fact proposed possible solutions for the unitarity problem [3-5], although the physical content of the theory is still not completely understood.

At the classical level, the Einstein-Weyl theory has recently attracted considerable interest. It is defined by the following action

$$
S=\int d^{4} x \sqrt{-g}\left(\gamma R-\alpha C_{\mu \nu \sigma \tau} C^{\mu \nu \sigma \tau}\right)
$$

where $C_{\mu \nu \sigma \tau}$ is the Weyl tensor. According to a generalized Israel theorem [6], any static black-hole solution of a generic quadratic gravity theory must have vanishing Ricci scalar $R$ in the exterior region (provided $\partial_{r} R$ goes to zero sufficiently rapidly at spatial infinity) and then be a solution of (1). This property has greatly simplified the search for black hole solution in quadratic gravity. In fact solutions with vanishing Ricci scalar and nonvanishing Ricci tensor $R_{\mu \nu}$ have been first discovered by [6] and further investigated in [7-9].

However, due to its strongly nonlinear nature, the general problem of connecting the weak-field regime with the strong one, is still not completely clarified. This is a central issue to discuss possible phenomenological and astrophysical implications of quadratic gravity. For instance if in the linearized theory the spin-2 mode is tachyonic as it emerges in the context of the asymptotic safety scenario [10,11], the Yukawa-like behavior $(1 / r) e^{ \pm m r}$ of the fields at large distances is turned into a periodic hair of the type $\sim(1 / r) \cos (|m| r)$ and the spacetime is no longer asymptotically flat. One would then like to know how the properties of the horizon (i.e., its location, surface gravity) and the further interior evolution, are determined by the asymptotic fields.

In this work, in order to tackle this problem, we employ a multiple shooting approach which allows a complete characterization of the solutions from $r=\infty$ down to $r=0$. Depending of the black hole "mass" $M$ defined at large distances we shall see that the thermal properties of the black holes and the behavior of the metric coefficient near $r=0$ are determined by the Yukawa coupling at infinity. On the contrary, for $m^{2}<0$ we discover a countable number of families of solutions, representing a new type of black hole. In particular the metric coefficient show characteristic ripples of wavelength $\sim 1 /|m|$ which resembles a gravitational analogous of the Friedel oscillations in plasma $[12,13]$.

\section{LINEARIZED SOLUTIONS AND NUMERICS}

Let us consider the field equations for (1)

$$
H_{\mu \nu}=R_{\mu \nu}-\frac{1}{2} R g_{\mu \nu}-\frac{2}{m^{2}}\left(\nabla^{\rho} \nabla^{\sigma}+\frac{1}{2} R^{\rho \sigma}\right) C_{\mu \rho \nu \sigma}
$$


where $m^{2}=\gamma / 2 \alpha$, and assume a static spherically symmetric spacetime of the form

$$
d s^{2}=-h(r) d t^{2}+\frac{d r^{2}}{f(r)}+r^{2} d \Omega^{2}
$$

Let us then write

$$
h(r)=1+V(r), \quad f(r)=1+W(r)
$$

with $V \ll 1$ and $W \ll 1$. The linearized field equations, describing the metric at large distances, can easily be obtained from the equations $H_{\mu}{ }^{\mu}=0$ and $H_{0}{ }^{0}-H_{i}{ }^{i}=0$, which reduce to

$$
\begin{aligned}
\nabla^{2}\left(\nabla^{2} V(r)+2 Y(r)\right) & =0 \\
\left(\nabla^{2}-\frac{3}{2} m^{2}\right) \nabla^{2} V(r)-\nabla^{2} Y(r) & =0
\end{aligned}
$$

where $Y(r)=r^{-2}(r W(r))^{\prime}$ (see [1] for a general discussion on the weak field expansion in quadratic gravity). The form of the linearized solution is

$$
\begin{aligned}
h(r)= & 1+C_{t}-\frac{2 M}{r}+2 S_{2-} \frac{\mathrm{e}^{-m r}}{r}+2 S_{2+} \frac{\mathrm{e}^{m r}}{r} \\
f(r)= & 1-\frac{2 M}{r}+S_{2-} \frac{\mathrm{e}^{-m r}}{r}(1+m r) \\
& +S_{2+} \frac{\mathrm{e}^{m r}}{r}(1-m r)
\end{aligned}
$$

where the dependence on the unknown $C_{t}, M, S_{2+}$ and $S_{2-}$ is explicit. Standard time parametrization at $r=\infty$ implies $C_{t}=0$ and asymptotically flat solutions have $S_{2+}=0$. The key question we are interested in is the determination of the values of $M$ and $S_{2-}$ for which a BH solution with a nonvanishing Ricci scalar is obtained. The trace of field equations (2) implies $R=0$ which, upon using the spherically symmetric ansatz Eq. (3), leads to the following second order equations:

$$
\begin{aligned}
0= & r h(r)\left(r f^{\prime}(r) h^{\prime}(r)+2 f(r)\left(r h^{\prime \prime}(r)+2 h^{\prime}(r)\right)\right) \\
& +4 h(r)^{2}\left(r f^{\prime}(r)+f(r)-1\right)-r^{2} f(r) h^{\prime}(r)^{2} .
\end{aligned}
$$

An additional second order equation can be obtained by considering a suitable combination [14]

$$
H_{r r}-X(r) H_{\mu}{ }^{\mu}-Y(r)\left(H_{\mu}{ }^{\mu}\right)^{2}-Z(r) \partial_{r} H_{\mu}{ }^{\mu}=0 .
$$

After some manipulations one obtains

$$
\begin{aligned}
0= & -r^{2} f(r) h(r)\left(r f^{\prime}(r)+3 f(r)\right) h^{\prime}(r)^{2} \\
& +2 r^{2} f(r) h(r)^{2} h^{\prime}(r)\left(-r f^{\prime \prime}(r)-f^{\prime}(r)+2 m^{2} r\right) \\
& +h(r)^{3}\left(r\left(-3 r f^{\prime}(r)^{2}+4 f^{\prime}(r)-4 m^{2} r\right)+r^{3} f(r)^{2} h^{\prime}(r)^{3}\right. \\
& \left.+4 f(r)\left(r^{2} f^{\prime \prime}(r)-r f^{\prime}(r)+m^{2} r^{2}+2\right)-8 f(r)^{2}\right) .
\end{aligned}
$$

It is not difficult to show that near a horizon $r_{H}$ the following local expansion holds

$f(r)=f_{1}\left(r-r_{H}\right)+f_{2}\left(f_{1}, r_{H}\right)\left(r-r_{H}\right)^{2}+\cdots$

$h(r)=h_{1}\left(\left(r-r_{H}\right)+h_{2}\left(f_{1}, r_{H}\right)\left(r-r_{H}\right)^{2}+\cdots\right)$

where $f_{1}$ and $h_{1}$ are two free parameters and $f_{i>1}, h_{i>1}$ are completely determined by $f_{1}$ and $r_{H}$, so that the Schwarzschild solution is obtained for $f_{1}=h_{1}=1 / r_{H}$. In particular the surface gravity is given by

$$
\kappa=\frac{1}{2} \sqrt{f_{1} h_{1}}
$$

and, as always, the Hawking temperature is $T=\kappa / 2 \pi$.

The space of the possible black hole solutions can be obtained by means of the following numerical strategy.

A weak field solution is assumed to be valid starting from some radius $r \gg 1 / m$, where the initial conditions (6) are set, and an inward numerical integration of (7)-(9) towards a fitting radius $r_{f}>r_{H}$ is performed. In particular, the rising Yukawa exponentials are switched off and $\left(M, S_{2-}\right)$ are assumed to be arbitrary. Moreover, $h_{1}$ and $f_{1}$ in (10) determine the initial condition for a corresponding outward integration towards $r_{f}$ from $r_{H}$. For actual calculations the Adaptive Stepsize Runge-Kutta integrator DO2PDF implemented by the NAG group (see https://www.nag.com for details) turned out to be rather efficient. Continuity at the fitting point of the functions $f(r), h(r)$ and their derivatives as a function of $\left(M, S_{2_{-}}, h_{1}, f_{1}\right)$ is obtained by means of a globally convergent Broyden's method as described in http://numerical.recipes. In particular we assumed a tolerance of $10^{-12}$ during the integration and a tolerance of $10^{-6}$ for the root finding algorithm. This method improves both the precision and the efficiency of finding $\mathrm{BH}$ solutions, in particular we find that the matching between the $\left(M, S_{2-}\right)$ and the $\left(h_{1}, f_{1}\right)$ parameters is improved of a factor $10^{4}$ compared to previous results.

The location of the fitting point can be changed in order to improve the numerical stability of the system, although our results do not depend on its precise location. It is convenient to set $m=1$ so that the radial coordinate $r$, and the constants $M, S_{2-}$ in Eq. (6) are all measured in units of $1 / \mathrm{m}$. By continuously changing the value of $r_{H}$ it is therefore possible to systematically explore the dependence of the asymptotic parameters of the solution on the parameters $h_{1}$ and $f_{1}$ of the local expansion near the horizon. Moreover, once the convergence is achieved, we 


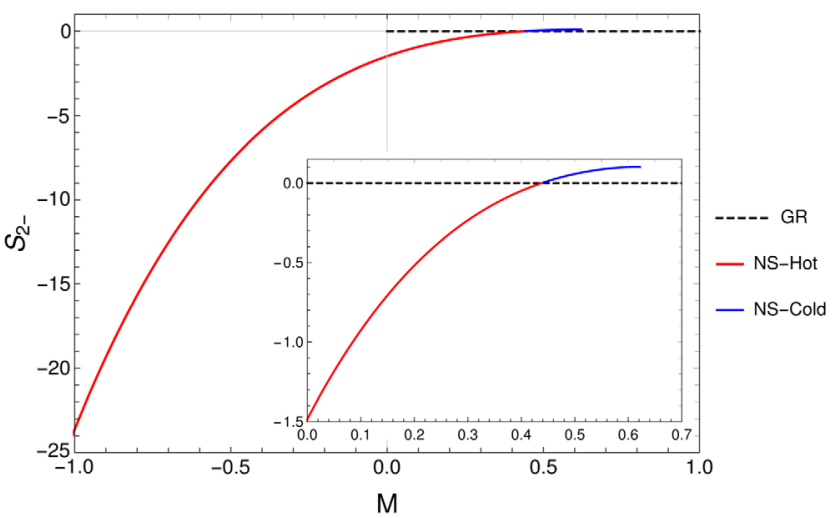

FIG. 1. Solutions space of non-Schwarzschild BHs. Red line indicates non-Schwarzshild black holes which are hotter than Schwarzshild black holes, blue line indicates colder black holes which are instead obtained for $S_{2-}>0$.

further shoot inward towards $r=0$ in order to characterize the behavior of the metric coefficients near the singularity. A single integration from $r \gg 1 / m$ to $r=0$ typically requires less than $10 s$, however the exploration of the full parameter space can take hours.

At last, the space of possible solutions is described in Figs. 1 and 2, where the inset on the right of Fig. 1 shows the region around $r_{H} \sim 0$. Black holes with $M>0$ only exist for $S_{2-}>-1.5$. Moreover, black holes with $S_{2-}>0$, represented with a blue line, are colder than the Schwarzschild black hole with the same horizon radius, while black holes with $S_{2-}<0$ are instead hotter, as shown in the inset of Fig. 2. If we compare the temperature of the non-Schwarzschild $\mathrm{BH}$ and the Schwarzschild ones with respect to the mass $M$, we find that the non-Schwarzschild ones are always colder. Black holes with large event horizon always have $M<0$. In the limit of zero temperature as $r_{H} \rightarrow 0$, the mass below $r_{H}<0.4$ assumes the constant value $M=M_{0}=0.62$.

\section{THE SINGULARITY AT $r=0$}

It is interesting to study the structure of the metric coefficient near $r=0$ as we move from the hot branch to the cold branch in Fig. 1. Therefore for each value of $r_{H}$ we further integrate toward $r=0$ in order to study the running exponents

$$
t=r \partial_{r} \ln h(r), \quad s=r \partial_{r} \ln f(r),
$$

which can be determined by stopping the numerical integration at a limiting value of the radius (we used $r=$ $10^{-6}$ in order to preserve numerical stability). The results are depicted in Fig. 3 where it can be noticed that as $r_{H}$ runs from the hot branch to the cold branch, $(s, t)$ run from $(-1,-1)$ to $(-2,2)$ (note that in our notation $s$ has the opposite sign of the one in [15]). The limiting configuration reached in the $r_{H} \rightarrow 0$ limit is the vanishing metric solution described in [16].

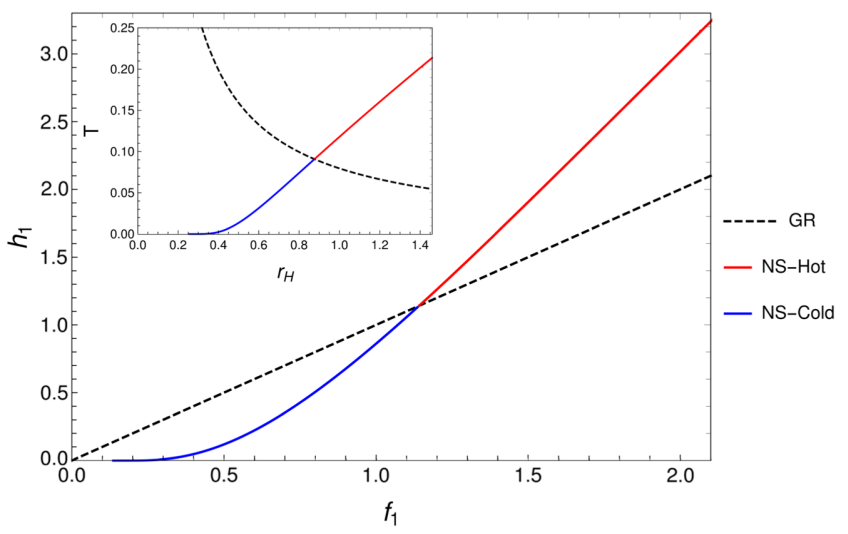

FIG. 2. Near-horizon parameters of the black hole solutions described in Fig. 1 and Hawking temperature.

To study this transition in detail let us define $x=-\ln r$ and rewrite Eq. (7) and Eq. (9) as a function of $s(x)$ and $t(x)$ in (12). Exploiting the fact that $f$ is large towards $r=0$ $(x=\infty)$ it is easy to obtain the following autonomous dynamical system

$\frac{d s}{d x}=-\frac{-2 s^{2} t+s^{2}-s t^{2}-8 s+t^{3}-3 t^{2}-8}{2(t-2)}$,

$\frac{d t}{d x}=-\frac{1}{2}\left(-s t-4 s-t^{2}-2 t-4\right)$.

Stationary solutions at $x=\infty$ determine the behavior of the metric near $r=0$. There are two fixed points [in addition to the trivial one $(0,0)], A=(-1,-1)$ which is an attractive improper node, and $B=(-2,2)$ which is an attractive node. Therefore as we move in the $\left(M, S_{2-}\right)$ plane, the asymptotic behavior near $r=0$ is completely described either by $A$ or $B$ in complete agreement with the Frobenius analysis in [15]. We find that around $r_{H}=0.86 \mathrm{a}$ transition occurs, as shown in Fig. 3, between a singular and a vanishing metric in the origin, in correspondence with

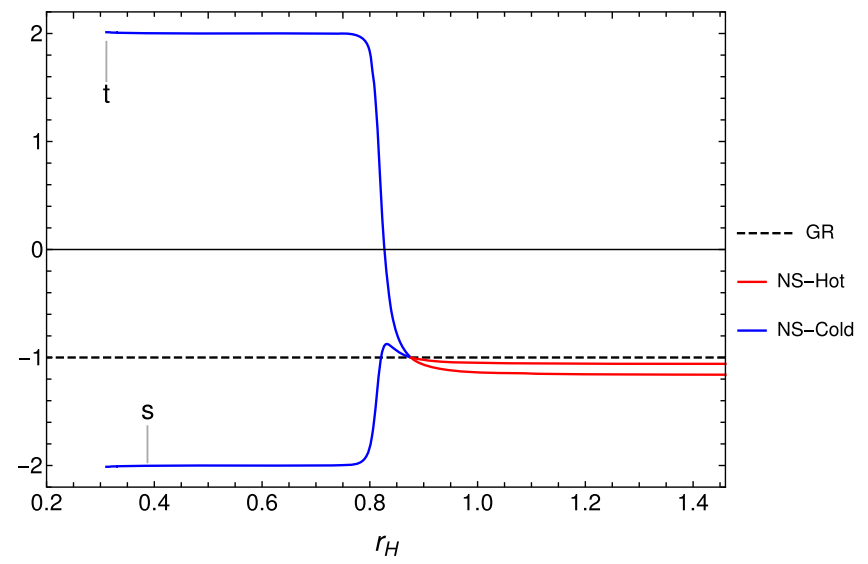

FIG. 3. Near-origin behavior of the black hole solutions described in Fig. 1. 

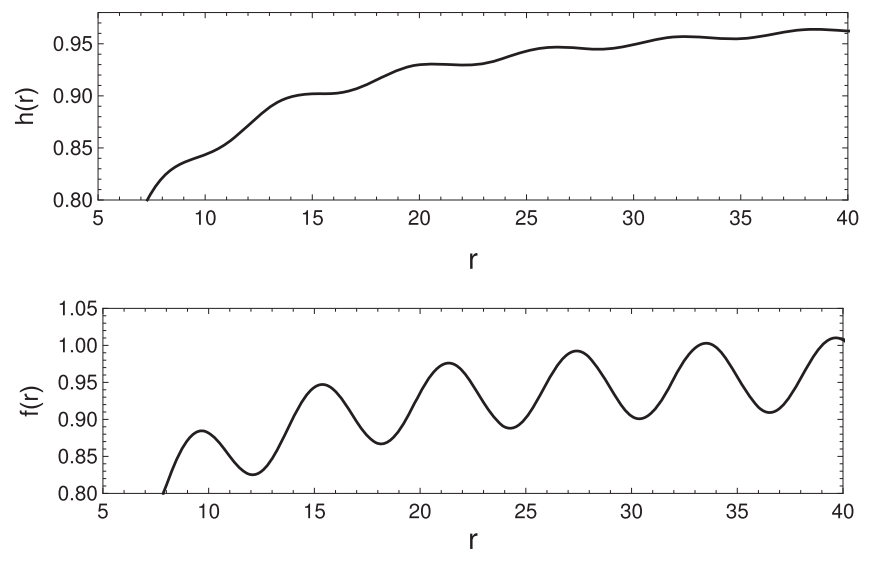

FIG. 4. A typical black hole solution for $m^{2}<0$. Notice the nonasymptotically flat behavior for large $r$ in the bottom panel. For this solution we have $A_{2}=-0.051$ and $M=0.746$.

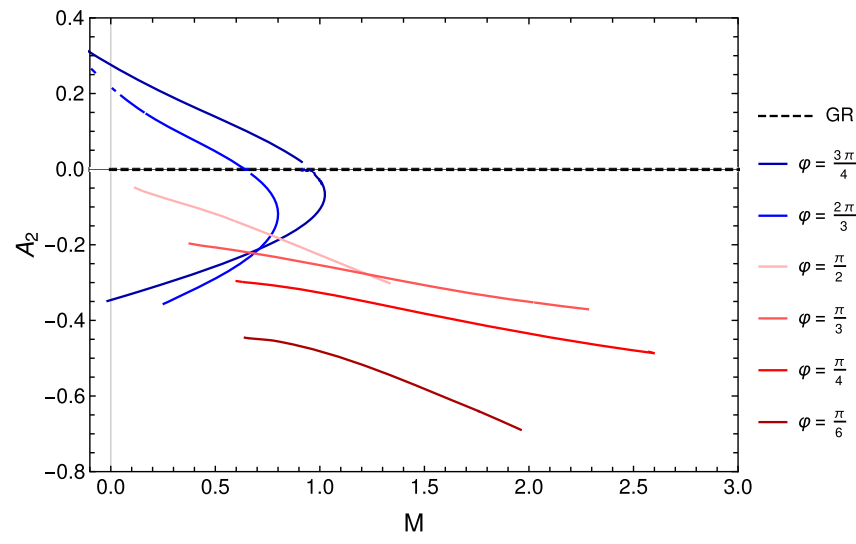

FIG. 5. Solutions space of non-Schwarzschild black holes for $m^{2}<0$, matching the weak-field expansion in Eq. (14) at large $r$ with $0<\varphi<\pi$, the solutions with $\pi<\varphi<2 \pi$ have the same $M$ but opposite $A_{2}$.

the transition between the hot and cold branch. Note however that, due to the improper node character of the point $\mathrm{A}$, the approach towards $(-1,1)$ is much slower, as displayed in Fig. 3.

\section{NON-SCHWARZSCHILD BLACK HOLE FOR $m^{2}<0$}

Motivated by recent results on the asymptotic safety scenario, we now consider the case $\alpha<0$ which implies that the spin-2 mass is imaginary. In this case the large distance expansion reads

$$
\begin{aligned}
h(r)= & 1+C_{t}-\frac{2 M}{r}+2 A_{2} \frac{\cos (|m| r+\varphi)}{r} \\
f(r)= & 1-\frac{2 M}{r}+A_{2} \frac{\cos (|m| r+\varphi)}{r} \\
& +A_{2}|m| \sin (|m| r+\varphi),
\end{aligned}
$$

which depends on four unknown constants (two coefficients $M$ and $A_{2}$, one phase $\varphi$, and the constant $C_{t}$ which we set to zero in the following). The spacetime is no longer asymptotically flat and we must require $A_{2}|m| \ll 1$ for our linearized solution to be valid at large values of the radial coordinate $r$.

Although spatial oscillations of this type have been discussed before in the framework of linearized gravity [13], in this work a complete solution is presented for the first time in the framework of Einstein-Weyl theory.

The shooting method used for the $m^{2}>0$ case can be applied also in this case. However, if in the ghost case we imposed asymptotic flatness at infinity and found a oneparameter family of solutions, in this case we leave the phase $\varphi$ as free parameter and we find a two-parameter family. This is a reflection of the ill-defined limit of (14) at large radii. A typical black hole solution is depicted in Fig. 4 where one can notice the large $r$-behavior of the metric coefficient according to (14).

If we assume the condition $A_{2} \ll 1$ a resulting space of possible solutions is depicted in Figs. 5 and 6. For the sake of clarity we show only some solutions with $0<\varphi<\pi$; any solution with $\varphi^{\prime}=\varphi+\pi$ has the same mass $M$, the same properties at the horizon but $A_{2}^{\prime}=-A_{2}$. Smaller mass $M$ corresponds to smaller horizon radius, except for $\pi / 2<\varphi<\pi$, where the mass reaches a maximum and then decreases for increasing radius. Moreover, for any fixed phase, the solutions have a maximum horizon radius where the parameters $f_{1}$ and $h_{1}$ vanish and diverge, respectively.

The solutions in blue are colder than the Schwarzschild solution with the same mass $M$, while the red ones are hotter; darker shades mean bigger differences between nonSchwarzschild and Schwarzschild temperatures. If we compare the temperatures in relation to the horizon radius, we find that BHs with $h_{1}<f_{1}$ are colder than the Schwarzschild ones, and the ones with $h_{1}>f_{1}$ are hotter. This phenomenon occurs for $A_{2}>0$ and $A_{2}<0$, respectively (for solutions with $0<\varphi<\pi$ ). In Fig. 6 we show the

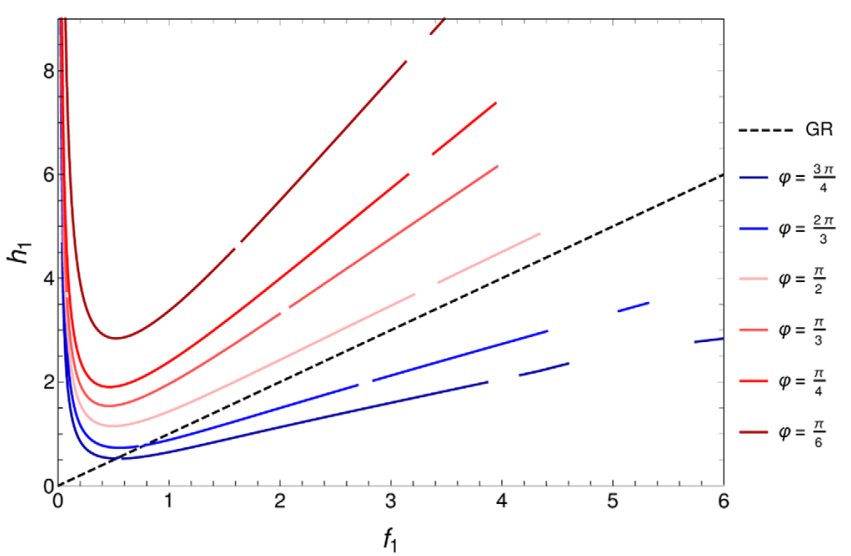

FIG. 6. Near-horizon parameters of non-Schwarzschild black holes for $m^{2}<0$ with $0<\varphi<\pi$; solutions with $\varphi^{\prime}=\varphi+\pi$ have the same near-horizon parameters. 
near-horizon parameter space, where increasing $f_{1}$ means decreasing $r_{H}$. It is interesting to note that the solutions populate almost the entire space, leading to a large variety in the thermodynamical properties of these black holes. The near-origin behavior of this non-Schwarzschild black holes is still not clear, given that the increasingly oscillating nature of the solutions makes the additional inward integration numerically unstable.

\section{CONCLUSIONS}

In this work we clarified some issues in the study of black holes in quadratic gravity. We managed to link the asymptotic metric at large distances with the series expansion around the horizon. With this characterization it will be possible to study both the gravitational and thermodynamical properties of these new black holes and how these properties affect each other. Moreover, we made a first attempt to numerically study the interior of these solutions, finding that the cold, Yukawa-repulsive black holes have a vanishing metric in the origin while the hot, Yukawaattractive ones have a singular metric.
For the first time we analyzed also the case where the parameter $\alpha$ is negative and then the massive spin- 2 field is tachyonic instead of ghostlike. We found, together with Schwarzschild BHs, nonasymptotically flat black hole solution. It is interesting to notice that this behavior resembles the rippled structure of the vacuum found in $R+R^{2}$ gravity due to the kinetic condensation of the conformal factor [17]. A detailed study of the gravothermodynamical properties of both ghostlike and tachyonic non-Schwarzschild black holes will be presented in further works.

\section{ACKNOWLEDGMENTS}

We would like to thank K. Stelle, A. Perkins, D. Litim and $\mathrm{H}$. Gies for many stimulating discussions. One of us would like to thank the INAF, Osservatorio Astrofisico di Catania for its hospitality, the Università di Milano Bicocca for financial support, and A. Tomasiello for his constant support and encouragement. We would also like to thank L. Santagati for a careful reading of the manuscript.
[1] K. S. Stelle, Phys. Rev. D 16, 953 (1977).

[2] M. Niedermaier and M. Reuter, Living Rev. Relativity 9, 5 (2006).

[3] D. Anselmi, J. High Energy Phys. 06 (2017) 086.

[4] D. Anselmi and M. Piva, J. High Energy Phys. 11 (2018) 21.

[5] D. Benedetti, P. F. Machado, and F. Saueressig, Mod. Phys. Lett. A 24, 2233 (2009).

[6] H. Lü, A. Perkins, C. N. Pope, and K. S. Stelle, Phys. Rev. Lett. 114, 171601 (2015).

[7] H. Lü, A. Perkins, C. N. Pope, and K. S. Stelle, Phys. Rev. D 92, 124019 (2015).

[8] K. Goldstein and J. J. Mashiyane, Phys. Rev. D 97, 024015 (2018).
[9] J. Podolský, R. Švarc, V. Pravda, and A. Pravdová, Phys. Rev. D 98, 021502(R) (2018).

[10] D. Benedetti, P. F. Machado, and F. Saueressig, Nucl. Phys. B824, 168 (2010).

[11] Y. Hamada and M. Yamada, J. High Energy Phys. 08 (2017) 70.

[12] J. Friedel, Adv. Phys. 3, 446 (1954).

[13] J. Boos, Int. J. Mod. Phys. D 27, 1847022 (2018).

[14] A. Perkins, Static spherically symmetric solutions in higher derivativs gravity, Ph.D. thesis, Imperial College, 2016.

[15] K. S. Stelle, Gen. Relativ. Gravit. 9, 353 (1978).

[16] B. Holdom and J. Ren, Phys. Rev. D 95, 084034 (2017).

[17] A. Bonanno and M. Reuter, Phys. Rev. D 87, 084019 (2013). 